\title{
First pathological study of canine primary breast lymphoma and the description of its clinicopathological characteristics as an animal model for human primary breast lymphoma
}

\author{
SANAZ RISMANCHI ${ }^{1}$, SAMAD MUHAMMADNEJAD ${ }^{2}$, SAEID AMANPOUR ${ }^{1}$, and AHAD MUHAMMADNEJAD ${ }^{3}$ \\ ${ }^{1}$ Cancer Models Research Center, Cancer Institute of Iran; ${ }^{2}$ Research Center for Molecular and Cellular Imaging; \\ ${ }^{3}$ Cancer Research Center, Cancer Institute of Iran, Tehran University of Medical Sciences, \\ Imam-Khomeini Hospital Complex, Tehran 1419733141, Iran
}

Received September 11, 2014; Accepted September 22, 2014

DOI: $10.3892 /$ br.2014.369

\begin{abstract}
Canine breast cancer (BC) and human BC are the most prevalent tumors in female dogs and humans, respectively. Several studies have indicated that canine BC is a good model for human BC. Unlike breast carcinomas, human primary breast lymphoma (PBL) is a rare tumor, but no case of canine PBL has been reported thus far. The current study presents a case of canine MC of the primary non-Hodgkin lymphoma (NHL) type for the first time and subsequently questions the theory of considering it as a model for human PBL. A $2-\mathrm{cm}$ tumor was surgically removed from the left caudal abdominal mammary gland of a 6-year-old female dog of the terrier breed. Microscopic examination did not show any sign for the epithelial origin of the tumor. By contrast, histomorphological view and molecular pathological evaluation by immunohistochemistry showed that the tumor was of the diffuse large B-cell lymphoma (DLBCL) type [cluster of differentiation $19^{+}$ $\left(\mathrm{CD}^{+} 9^{+}\right), \mathrm{CD} 20^{+}, \mathrm{CD}^{+} 0^{+}$, B-cell lymphoma 6+, CD3-, CD15According to the World Health Organization classification, DLBCL is considered to be an NHL. Canine NHL is common in dogs and certain investigators believe that the biological behavior and clinical course is extremely similar to human NHL, and therefore, consider it as a model of human NHL. To the best of our knowledge, the current study is the first report of canine PBL. As the most significantly reported human PBL histotype is the DLBCL type, the histomorphological and immunophenotyping characteristics of canine PBL in the study considerably match with human PBL and raise the hypothesis that it can be a model for human PBL.
\end{abstract}

Correspondence to: Dr Ahad Muhammadnejad, Cancer Research Center, Cancer Institute of Iran, Tehran University of Medical Sciences, Imam-Khomeini Hospital Complex, Keshavarz Blvd, Tehran 1419733141, Iran

E-mail: mohamadnajad@yahoo.com

Key words: breast neoplasms, animal mammary neoplasms, non-Hodgkin lymphoma, diffuse large B-Cell lymphoma

\section{Introduction}

Canine breast cancer (BC) is the most significant tumor in female dogs, whereas human $\mathrm{BC}$ is the most prevalent type of cancer in females worldwide (1-2). There is much evidence indicating that canine $\mathrm{BC}$ mimics the characteristics of human $\mathrm{BC}$ with regards to incidence, histomorphological characteristics, gene profiles, molecular signaling pathways and clinical course (3). Numerous experts believe that canine BC can be a powerful translation research model for human BC (4). Carcinomas account for the majority of histological classifications of canine BC and sarcomas are less frequent (5). However, to the best of our knowledge no case of canine primary lymphoma of the breast has been reported. However, human primary breast lymphoma (PBL) is a rare malignancy and statistics show that $<0.5 \%$ of human BCs and $2 \%$ of all extranodal non-Hodgkin lymphomas (NHLs) are PBL (6).

The current study presents a case of canine $\mathrm{BC}$ of the primary NHL type for the first time and subsequently the theory of considering it as a model for human PBL is raised.

\section{Materials and methods}

A 6-year-old female terrier with a history of ovariohysterectomy at the age of 1 was referred to a veterinary clinic with a lump in the left caudal abdominal mammary gland. The primary diagnosis of the veterinary surgeon was canine BC. Thoracic imaging and abdominal ultrasonography (according to the diagnostic guidelines of metastatic canine BC) were performed to check for metastasis, but no symptom was observed (7). Finally, simple mastectomy was performed and a sample of the 2-cm tumor was sent to the pathology laboratory of cancer institute of Iran.

The tumor section was fixed by $4 \%$ formaldehyde in $0.1 \mathrm{M}$ phosphate-buffered saline (PBS) solution. The fixed tissue was dehydrated by graded concentrations of ethanol and embedded in paraffin wax, and subsequently stained with hematoxylin and eosin (H\&E). The slides were reviewed by a pathologist. For immunohistochemical examination, paraffin-embedded blocks were cooled in an ice-water mixture for $30 \mathrm{~min}$ before sectioning. A total of $4 \mu \mathrm{m}$ sections were cut and placed on 
Table I. Immunohistochemistry (IHC) test results assessed by CD markers.

\begin{tabular}{ll}
\hline IHC staining & CD marker result \\
\hline $\mathrm{CD} 3$ & Negative \\
$\mathrm{CD} 10$ & Positive \\
$\mathrm{CD} 15$ & Negative \\
$\mathrm{CD} 19$ & Positive \\
$\mathrm{CD} 20$ & Positive \\
Bcl-6 & Scatter \\
\hline
\end{tabular}

$\mathrm{CD}$, cluster of differentiation; Bcl-6, B-cell lymphoma 6.

slides. After a brief drying period of $\sim 15 \mathrm{~min}$, the sections were heat-fixed to the slide at $37^{\circ} \mathrm{C}$. The sections were deparaffinized and rehydrated in graded ethanol concentrations. The prepared slides were stained immunohistochemically by cytokeratin 7 (CK7), CK5/6, cluster of differentiation 3 (CD3), CD10, CD15, CD19, CD20, and Bcl-6 according to the manufacturer's instructions for the kits (Dako Denmark A/S, Glostrup, Denmark).

\section{Results}

No cytology or histology assessments were performed prior to the surgery. No evidence of carcinoma was observed during the microscopic study of H\&E slides, and the arrangement of malignant cells indicated lymphoproliferative neoplasms.
Immunohistochemistry (IHC) staining with CK7 and CK5/6 markers confirmed that the tumor did not have an epithelial nature. Prominent histomorphological characteristics of the malignant cells are described as follows: Cell sizes were larger than normal lymphocytes and they had round and elliptical vesicular nuclei. Nucleoli were often isolated and they were observed near the nuclear membrane (Fig. 1). The cytoplasm of the cells was often basophilic and certain anaplastic cells appeared to be multinucleate and showed the pathological view of Reed-Sternberg-like cells (Fig. 1). According to histomorphological characteristics, the pattern of malignant cells showed diffuse large B-cell lymphoma (DLBCL), however, the Reed-Sternberg-like cells view was misleading and could show a Hodgkin's lymphoma (HL) pattern. In order to determine the immunophenotype of tumor cells, slides were prepared from paraffin blocks and subsequently stained with the IHC method using CD3, CD10, CD15, CD19, CD20 and B-cell lymphoma 6 markers (Fig. 1). IHC results are observed in Table I. In addition, Ki-67 marker staining showed that cell cycle activity was $\sim 50 \%$ and could reject the possibility of Burkitt's lymphoma (starry sky pattern was not observed with regards to histomorphology).

With regard to the histomorphological findings and IHC, the final diagnosis was extranodal-NHL of the DLBCL type and considering the clinical findings that showed no evidence of a primary lymphoma having invaded the breast area, the final diagnosis for this case was canine PBL.

\section{Discussion}

As it was indicated, the animal had canine $\mathrm{BC}$ of the primary NHL type. Canine NHL is a common malignancy (8). However,
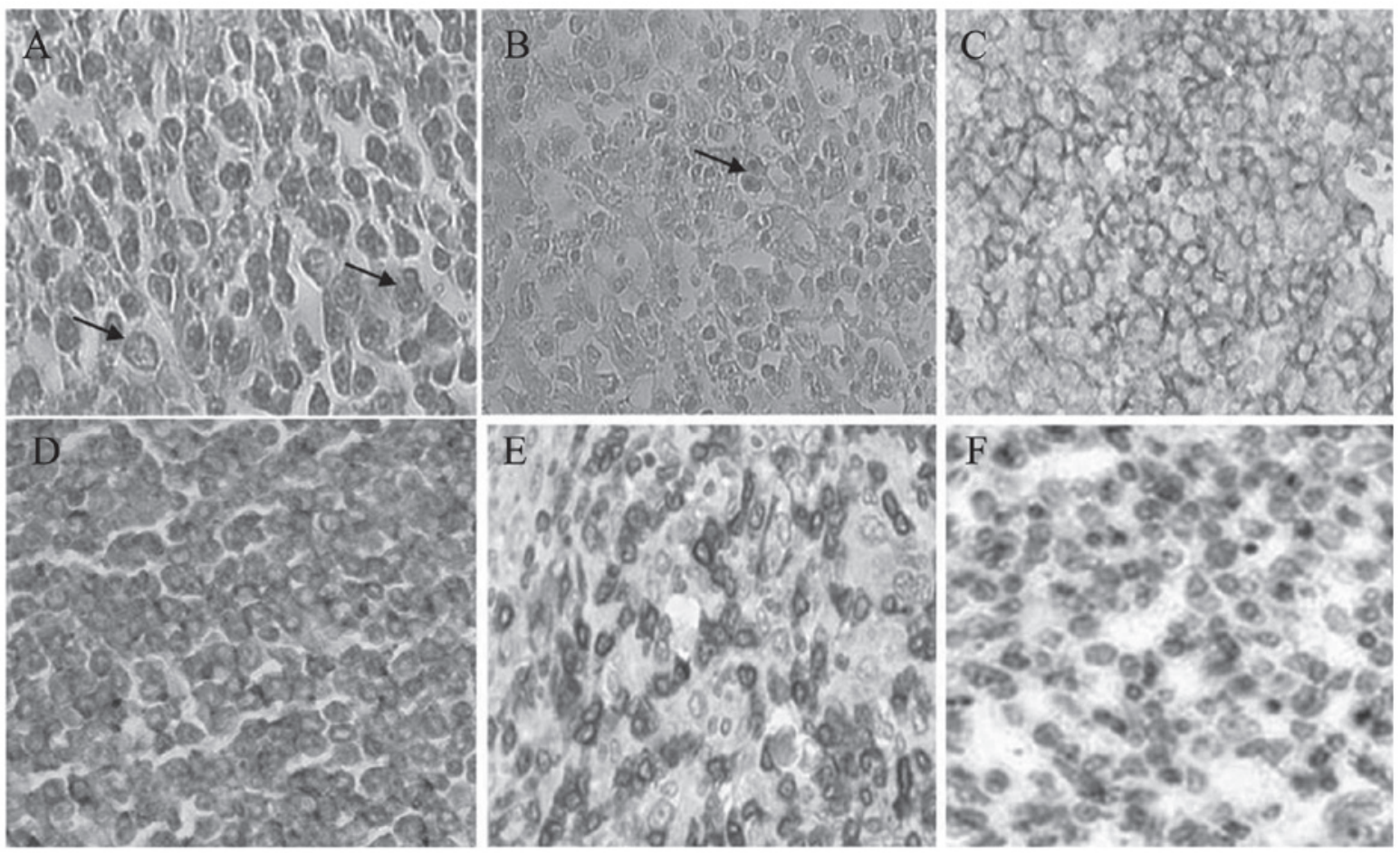

Figure 1. Micrographs prepared from canine primary breast lymphoma. (A and B) Micrographs show hematoxylin and eosin-stained slides. Large lymphocyte cells with vesicular nuclei were observed. The tip of the arrow in micrograph (B) shows Reed-Sternberg-like cells. (C-F) Micrographs show immunohistochemistry staining. (C) Marker CD10 was positive, (D and E) CD19 and CD20 were positive, respectively, and (F) shows the surface marker of Bcl-6 in the form of scatter. CD, cluster of differentiation, Bcl-6, B-cell lymphoma 6. 
this disease comprises a heterogeneous group of canine malignancies and has an extremely varied prognosis (9). The major cause is attributed to various NHL subtypes. Canine NHL subtypes were examined with immunophenotyping assessments, and considering their immunophenotyping similarities and adaptations with human NHL, canine NHL was classified according to World Health Organization guidelines (10). DLBCL comprises the most significant group of canine NHL, as statistics show that $48 \%$ of all canine lymphomas belong to this category (11). Extensive evidence suggests canine NHL to be a model for human NHL. Following the separation of canine lymphoma cells, the study by Ito et al (8) cultured and passaged them and developed a xenograft model. Subsequently, their molecular profile was examined and it was concluded that not only is canine NHL morphologically and behaviorally similar to human NHL, but its molecular changes also mimic human lymphoma. Pawlak et al (10) had previously addressed this theory.

In human DLBCL it is known that tumor growth is rapid and prognosis is poor $(12,13)$. Bienzle and Vernau (9) stated that the survival time of canine DLBCL is short, and that similar to human DLBCL, its prognosis is bad. Using molecular techniques, Richards et al (11) divided canine DLBCL into two subcategories. After assessing their survival time, the study concluded that the course of the disease is similar to human DLBCL and its prognosis is poor.

Human PBL is a relatively rare form of human BC and the majority of its types are DLBCL (6). No previous study of canine breast lymphoma can be found in the databases of PubMed and Google scholar. However, the histomorphological characteristics, molecular pathology and clinical data of the present case confirmed breast lymphoma. Observation of Reed-Sternberg-like cells may be suggestive of canine HL, but the surface marker of CD15 was negative. Such a microscopic view appears to be due to the presence of large inclusion-like nucleoli in highly anaplastic cells, whereas this surface marker is positive in $>90 \%$ of typical Reed-Sternberg cells (14). The results of the present case report indicate that canine NHL in the mammary gland area can mimic the properties of HBL.

In general, modeling in the area of oncology research requires reliable evidence showing that the model is powerful regarding translational research, as the results of the pre-clinical phase require extension to the clinical phase (15). Although reliable scientific communities have suggested xenograft for the pre-clinical phase of the tumor, the canine model has received increasing attention in oncology research during recent years $(3,10)$. Three main causes have been cited for this: Firstly, these tumors are spontaneous, as they were not experimentally induced; secondly, the life of dogs are so that the clinical course of the disease is extremely well shown and the disease shifts from early to advance stage. As a result, invasion and metastasis can be followed in this model. Thirdly, with regard to the fact that dogs live alongside humans, they are exposed to the same risk factors and so the course of molecular changes and genetic mutations can also be studied $(4,8,15)$.

Although no studies of canine breast lymphoma have been reported thus far, and to the best of our knowledge, this is the first report of canine NHL in the mammary area of dog, when considering previous evidence emphasizing the similarities in histomorphology, immunophenotyping and clinical course of canine and human NHL, the theory can be raised that canine PBL can also be a model for research on human PBL. Performing case series studies in this area in the future is required. Not only can canine cancer models be considered a study phase in pre-clinical research, but they can also be useful for afflicted dogs, as they may be helpful in developing novel (ethical) treatments and also reduce the suffering caused by canine cancer.

\section{Acknowledgements}

The present study had no financial sponsor and all the costs of pathology tests were paid by the authors. The authors would like to express their gratitude to Dr Taghizadeh-jahed, veterinary surgeon, for surgically removing the tumor. They would also like to thank Ms. Morsali and the Pathobiology Laboratory of Dr E'temad Moghaddam for helping prepare the pathological slides.

\section{References}

1. Sleeckx N, de Rooster H, Veldhuis Kroeze EJ, Van Ginneken C and Van Brantegem L: Canine mammary tumours, an overview. Reprod Domest Anim 46: 1112-1131, 2011.

2. Redig AJ and McAllister SS: Breast cancer as a systemic disease: a view of metastasis. J Intern Med 274: 113-126, 2013.

3. Pinho SS, Carvalho S, Cabral J, Reis CA and Gartner F: Canine tumors: a spontaneous animal model of human carcinogenesis. Transl Res 159: 165-172, 2012.

4. Rivera $\mathrm{P}$ and von Euler H: Molecular biological aspects on canine and human mammary tumors. Vet Pathol 48: 132-146, 2011.

5. Goldschmidt M, Peña L, Rasotto R and Zappulli V: Classification and grading of canine mammary tumors. Vet Pathol 48: 117-131, 2011.

6. Cheah CY, Campbell BA and Seymour JF: Primary breast lymphoma. Cancer Treat Rev 40: 900-908, 2014.

7. Cassali GD, Lavalle GE, De Nardi AB, et al: Consensus for the diagnosis, prognosis and treatment of canine mammary tumors. Braz J Vet Pathol 4: 153-180, 2011.

8. Ito D, Frantz AM and Modiano JF: Canine lymphoma as a comparative model for human non-Hodgkin lymphoma: recent progress and applications. Vet Immunol Immunopathol 159: 192-201, 2014.

9. Bienzle D and Vernau W: The diagnostic assessment of canine lymphoma: implications for treatment. Clin Lab Med 31: 21-39, 2011.

10. Pawlak A, Obminska-Mrukowicz B and Rapak A: The dog as a model for comparative studies of lymphoma and leukemia in humans. Postepy Hig Med Dosw (Online) 67: 471-480, 2013 (In Polish).

11. Richards KL, Motsinger-Reif AA, Chen HW, et al: Gene profiling of canine B-cell lymphoma reveals germinal center and postgerminal center subtypes with different survival times, modeling human DLBCL. Cancer Res 73: 5029-5039, 2013.

12. Aviv A, Tadmor T and Polliack A: Primary diffuse large B-cell lymphoma of the breast: looking at pathogenesis, clinical issues and therapeutic options. Ann Oncol 24: 2236-2244, 2013.

13. Roschewski M, Dunleavy K and Wilson WH: Diffuse large B cell lymphoma: molecular targeted therapy. Int J Hematol 96: 552-561, 2012.

14. Hansmann ML and Willenbrock K: WHO classification of Hodgkin's lymphoma and its molecular pathological relevance. Pathologe 23: 207-218, 2002 (In German).

15. Vail DM and MacEwen EG: Spontaneously occurring tumors of companion animals as models for human cancer. Cancer Invest 18: 781-792, 2000. 